\title{
Neoadjuvant Treatment of Breast Cancer - Advances and Limitations
}

\author{
Peter A. Fasching Paul Gaß Alexander Hein \\ Department of Gynecology and Obstetrics, University Hospital Erlangen, Comprehensive Cancer Center Erlangen-EMN, \\ Friedrich-Alexander University Erlangen-Nuremberg, Erlangen, Germany
}

Neoadjuvant chemotherapy was first introduced for the treatment of larger tumors in order to reduce tumor size and possibly increase the likelihood for a breast conserving therapy; however, after decades of clinical use of neoadjuvant chemotherapy, it can be considered as a standard chemotherapy option for any patient who has an indication for neoadjuvant chemotherapy $[1,2]$.

In addition to a possible clinical benefit by downstaging the tumor before surgery, a great interest in neoadjuvant chemotherapy has been developed because it serves as an in vivo experiment for both patients and physicians to see whether or not the tumor responds to the given therapy. In a relevant number of patients the tumor completely disappears from breast and the axilla. It has been shown that this pathological complete response (pCR) is an excellent predictor of prognosis, especially in some molecular subgroups $[3,4]$.

In a pooled analysis, up to $35 \%$ of all patients with triple negative breast cancer (TNBC) achieved pCR after neoadjuvant chemotherapy, while in HER2-positive hormone receptor (HR)-negative patients up to $50 \%$ achieved pCR after chemotherapy and trastuzumab (TCH) therapy [3]. Much lower pCR rates are achieved in HR-positive, HER2-negative patients, with pCR rates as low as $8 \%$ for tumors with a grading of 1 or 2 and about $16 \%$ with a grading of 3 [3].

With regard to outcomes, the translation of pCR into a good prognosis is prominent for triple negative and HER2-positive patients; however, this is difficult to show for other subtypes, such as HR-positive patients with a grading of 1 and 2 [3]. In this issue of Breast CARE, Gaß et al. [5] explore which HR-positive patients should receive adjuvant and neoadjuvant chemotherapy. The treatment of these patients is a challenge because they have a good prognosis but often do not respond to chemotherapy. Novel molecular tests might help to identify those patients who have an excellent prognosis and therefore do not need chemotherapy $[6,7]$. The low response rates in HR-positive, HER2-negative patients has led to a focus of neoadjuvant chemotherapy in triple negative and HER2-positive patients, with some studies only enrolling those 2 patient groups [8, 9].

Knowing that patients with some tumor types more often achieve pCR than others and that the connection between pCR and prognosis differs between subgroups, one recent focus of research is to differentiate patients who benefit from pCR from those in which a complete disappearance of the tumor does not have a larger prognostic effect. One example is the subgroup of triple negative patients with a BRCA mutation: in a clinical cohort treated neoadjuvantly with anthracyclines and taxanes a prognostic effect of pCR could only be shown in patients with a wildtype BRCA status [10]. Similarly, patients with a PIR3K mutation in their tumor often achieve a pCR after neoadjuvant chemotherapy combined with anti-HER2 therapy. However, a PI3K mutation does not seem to have influence on prognosis [11]. This shows that we still have a lot to learn concerning the connection between pCR and prognosis.

It was tried to establish pCR as a surrogate marker for prognosis, which could lead to the approval of neoadjuvant therapies. However, the combined analysis of almost 12,000 patients from 12 international trials did not validate $\mathrm{pCR}$ as a surrogate marker for prognosis [3]. In a subgroup analysis, the correlation between pCR and prognosis was largest in HER2-positive patients [3, 12], indicating that further research on how to use response to neoadjuvant therapy for drug development might be most successful in this breast cancer subtype. In this issue of BREAST CARE, Kolberg and colleagues [13] present the 4-year follow-up of patients who achieved pCR after neoadjuvant treatment with a combination of docetaxel, carboplatin, and weekly TCH.

Patients who have residual disease after neoadjuvant chemotherapy are a clinical problem, as they represent a subgroup with very unfavorable prognosis. Novel therapy concepts consider these patients for an additional adjuvant therapy in so-called post-

\section{KARGER}

() 2016 S. Karger GmbH, Freiburg
Prof. Dr. med. Peter A. Fasching

Department of Gynecology and Obstetrics, University Hospital Erlangen

Comprehensive Cancer Center Erlangen-EMN, Friedrich-Alexander University Erlangen-Nuremberg

Universitätsstraße 21-23, 91054 Erlangen, Germany

Peter.Fasching@uk-erlangen.de 
neoadjuvant studies. Examples for those studies are the Katherine (NCT01772472), Penelope-B (NCT01864746) and Olympia (NCT02032823) trials.

It must be our goal for the next years to develop neoadjuvant chemotherapy to a reliable model that connects an increase of $\mathrm{pCR}$ with prognosis. Over the last years it could be shown that molecular subtypes might play a role in this connection. Therefore, biomarker research is one of the most promising fields in neoadjuvant chemotherapy research. The possibility to collect tumor samples in the course of therapy and compare molecular profiles before, during, and after chemotherapy might reveal resistance mechanisms and pathways which predict a high therapy response. A good prediction of pCR can already be achieved combining estrogen receptor status, progesterone receptor status, HER2, and Ki-67 [14, 15]. However, these markers have been available for decades. Modern molecular analyses now integrate all levels of systems biology, including analyses of tumor mutations, inherited genetic variants, epigenetic changes, microRNA, and proteins as well as their interactions in metabolic, signal transduction and further pathways. The integration of these analyses in the clinical context of neoadjuvant chemotherapy will be a challenge as sample size is an important issue with regard to many biomarkers, which need not only discovery but also validation for clinical use. The global harmonization and interconnection of clinical trial networks will also be one major challenge over the next few years. However, this is necessary to advance knowledge in neoadjuvant breast cancer research

\section{Disclosure Statement}

PAF carried out research for Novartis and Amgen. PG and AH declare that there are no conflicts of interest.

\section{References}

1 Kaufmann M, Hortobagyi GN, Goldhirsch A, et al. Recommendations from an international expert panel on the use of neoadjuvant (primary) systemic treatment of operable breast cancer: an update. J Clin Oncol 2006;24:1940-1949.

2 Arbeitsgemeinschaft Gynakologische Onkologie Kommission Mamma: Diagnosis and Treatment of Patients with Primary and Metastatic Breast Cancer Guidelines of the AGO Breast Committee. www. ago-online.de/fileadmin/downloads/leitlinien/mamma/ Maerz2016/de/AGO_englisch_deutsch_gesamt.zip 2016;accessed October 11, 2016.

3 Cortazar P, Zhang L, Untch M, et al.: Pathological complete response and long-term clinical benefit in breast cancer: the CTNeoBC pooled analysis. Lancet 2014;384:164-172.

4 von Minckwitz G, Untch M, Blohmer JU, et al.: Definition and impact of pathologic complete response on prognosis after neoadjuvant chemotherapy in various intrinsic breast cancer subtypes. J Clin Oncol 2012;30 1796-1804.

5 Gaß P, Fasching PA, Fehm T, et al.: Factors influencing decision-making for or against adjuvant and neoadjuvant chemotherapy in postmenopausal hormone receptor-positive breast cancer patients in the EvAluateTM study. Breast Care 2016;DOI: 10.1159/000452468.
6 Sparano JA, Gray RJ, Makower DF, et al.: Prospective validation of a 21-gene expression assay in breast cancer. N Engl J Med 2015;373:2005-2014.

7 Cardoso F, van't Veer LJ, Bogaerts J, et al.: 70-Gene signature as an aid to treatment decisions in earlystage breast cancer. N Engl J Med 2016;375:717-729.

8 Untch M, Jackisch C, Schneeweiss A, et al.: Nab-paclitaxel versus solvent-based paclitaxel in neoadjuvant chemotherapy for early breast cancer (GeparSeptoGBG 69): a randomised, phase 3 trial. Lancet Oncol 2016; 17:345-356.

9 von Minckwitz G, Schneeweiss A, Loibl S, et al.: Neoadjuvant carboplatin in patients with triple-negative and HER2-positive early breast cancer (GeparSixto; GBG 66): a randomised phase 2 trial. Lancet Oncol 2014;15:747-756.

10 Paluch-Shimon S, Friedman E, Berger R, et al.: Neoadjuvant doxorubicin and cyclophosphamide followed by paclitaxel in triple-negative breast cancer among BRCA1 mutation carriers and non-carriers. Breast Cancer Res Treat 2016;157:157-165.
11 Loibl S, von Minckwitz G, Schneeweiss A, et al. PIK3CA mutations are associated with lower rates of pathologic complete response to anti-human epidermal growth factor receptor 2 (HER2) therapy in primary HER2-overexpressing breast cancer. J Clin Oncol 2014;32:3212-3220.

12 Gianni L, Pienkowski T, Im YH, et al.: 5-year analysis of neoadjuvant pertuzumab and trastuzumab in patients with locally advanced, inflammatory, or earlystage HER2-positive breast cancer (NeoSphere): a multicentre, open-label, phase 2 randomised trial. Lancet Oncol 2016;17:791-800

13 Kolberg H-C, Akpolat-Basci L, Stephanou M, et al.: Neoadjuvant chemotherapy with docetaxel, carboplatin and weekly trastuzumab is active in HER2-positive early breast cancer: results after a median follow-up of over 4 years. Breast Care 2016;DOI: 10.1159/000452079.

14 Fasching PA, Heusinger K, Haeberle L, et al.: Ki67, chemotherapy response, and prognosis in breast cancer patients receiving neoadjuvant treatment. BMC Cancer 2011;11:486.

15 von Minckwitz G, Schmitt WD, Loibl S, et al.: Ki67 measured after neoadjuvant chemotherapy for primary breast cancer. Clin Cancer Res 2013;19:4521-4531. 